\title{
Humidity Sensing Properties of Chitosan by Using Quartz Crystal Microbalance Method
}

\author{
A. Kemal Havare ${ }^{1,3}$, Hüseyin İlgü², Salih Okur ${ }^{3, *}$, and Gülşah Şanlı-Mohamed ${ }^{2, *}$ \\ ${ }^{1}$ Institute of Solar Energy, Bornova, Ege University, Izmir, 35100, Turkey \\ ${ }^{2}$ Department of Chemistry, izmir Institute of Technology, Urla, Izmir, 35430, Turkey \\ ${ }^{3}$ Departement of Material Science and Engineering, Izmir Katip Çelebi University, 35620 Çiğli, İmir, Turkey, Turkey
}

(Received: 24 December 2011. Accepted: 18 March 2012)

\begin{abstract}
Humidity adsorption kinetics of chitosan films was investigated by quartz crystal microbalance (QCM) technique. In this study, chitosan was synthesized from chitin by deacetylation process in $2.0 \%(\mathrm{v} / \mathrm{v})$ acetic acid solution and then coated on QCM to measure humidity response. The Langmuir model was used to determine the adsorption rates and Gibbs free energy for various relative humidity between $11 \%$ and $94 \%$. The average Gibbs free energy for adsorption was obtained as $-12.93 \mathrm{~kJ} / \mathrm{mol}$. Our reproducible experimental results show that chitosan films are very sensitive to relative humidity changes at room temperature.
\end{abstract}

Keywords: Chitosan, Humidity Sensing, Langmuir Model, Adsorption, Desorption.

\section{INTRODUCTION}

Controlling humidity is very important issue in production and manufacturing process such as food, ${ }^{1}$ automotive, ${ }^{2}$ electronics, and agriculture ${ }^{3}$ industries. Therefore, reliable, cheap, and highly sensitive humidity detectors are necessary. There are many types of humidity sensors based on ceramic, ${ }^{4}$ carbon based materials, ${ }^{5-7}$ polymeric, ${ }^{8-11}$ and composite materials. ${ }^{12}$ Ceramic sensors need an integrated heater to recover their humidity-sensitive characteristics, while polymeric sensors do not need a heating process, but they have been found to be less sensitive. ${ }^{11}$ In recent years, the sensing properties of humidity sensors have been enhanced significantly ${ }^{13}$ due to developing novel functional sensing elements such as organic calix[4]arene ${ }^{14}$ and organometallic Ruthenium ${ }^{15}$ derivatives with carboxylate and sulphonate groups.

Here, we have investigated the humidity sensing capability of drop-casted chitosan films with functional amine and hydroxyl groups in the molecular structure prepared in acetic acid solution. Chitosan is a biocompatible material and because of its characteristics, it has attracted so much attention of many scientists and has been used in a variety of areas especially for gene delivery, enzyme immobilization, drug targeting and so on. Chitosan is

\footnotetext{
*Corresponding authors; E-mails: salihokur@iyte.edu.tr,
} gulsahsanli@iyte.edu.tr a linear polysaccharide composed essentially of $\beta-(1-4)$ linked glucosamine units together with some proportion of $N$-acetyl glucosamine units. It is generally obtained by $N$-deacetylation of chitin to a varying extent.

Quartz Microbalance (QCM) technique comprises a thin vibrating AT-cut quartz wafer sandwiched between two metal excitation electrodes. QCM is highly sensitive to mass changes $\sim 1 \mathrm{ng} / \mathrm{sq}-\mathrm{cm}$. The performance characteristics of the QCM-based sensor depend on the chemical nature and physical properties of the coating material. ${ }^{8}$

QCM is based on frequency shift of coated quartz crystal with sensing element due to adsorption of humidity atoms on the surface of the sensing material. ${ }^{16}$ The mass change $(\Delta m)$ on surface of the quartz crystal is calculated by using Sauerbrey Eq. (7) from the frequency change $(\Delta m)$

$$
\Delta m=-\frac{A \sqrt{\mu \rho}}{2 f_{0}^{2}} \times \Delta f=-C \times \Delta f
$$

where $f_{0}$ is the resonant frequency of the fundamental mode of the QCM crystal, $A$ is the area of the gold disk coated onto the crystal, $\rho$ is the density of the crystal, and $\mu$ is the shear modulus of quartz. Thus the frequency shift is directly proportional to the adsorbed mass on the chitosan modified gold QCM electrodes. ${ }^{17}$ 


\section{EXPERIMENTAL DETAILS}

\subsection{Synthesis and Film Preparation of Chitosan}

The chemicals; chitin and sodium tripolyphophate pentabasic were purchased from Sigma; sodium hydroxide anhydrous and acetic acid glacial were purchased from Carlo Erba. All solutions were prepared using distilled water. Chitosan was synthesized from chitin via deactylation by using readily reported method of Rigby and Wolfrom with some modification described by Boyac $\iota$ et al. ${ }^{18}$ Briefly, $15.0 \mathrm{~g}$ of chitin was heated in $720.0 \mathrm{ml}$ of $40.0 \%(\mathrm{w} / \mathrm{w})$ aqueous $\mathrm{NaOH}$ solution at $115^{\circ} \mathrm{C}$ for $6 \mathrm{~h}$ under nitrogen atmosphere. The chitosan was washed with distilled water until the $\mathrm{pH}$ reaches neutrality and dried at $60{ }^{\circ} \mathrm{C}$ for $2 \mathrm{~h}$. Figure 1 shows the chemical structure of chitosan with some functional groups, such as amino group, at two different positions and hydroxyl groups as good hydrogen bond donor and acceptor.

Chitosan in $2.0 \%(\mathrm{v} / \mathrm{v})$ acetic acid solution was coated by drop casting technique on gold coated QCM electrodes. After drying at room conditions, it was kept in desiccators at room temperature for $3 \mathrm{~h}$. FT-IR analysis of chitin and chitosan were done with $\mathrm{KBr}$ pellet, ratio of 1:100 (sample: sample $+\mathrm{KBr}$ ). The FT-IR results are given in Figure 2 and are highly compatible with the previous works in literature. ${ }^{19-21}$ Scanning electron microscopy (SEM) was used to investigate the morphology of chitosan.

\subsection{Humidity Measurement Technique}

A time-resolved electrochemical quartz crystal microbalance (EQCM) with the model of CHI400A Series from $\mathrm{CH}$ Instruments (Austin, USA) has been used to measure the changes in the resonance frequency of quartz crystals between gold electrodes via both serial and USB interface connected to a computer. The QCM works with oscillation frequencies between 7.995 MHz and 7.950 MHz. The density $\rho$ of the crystal is $2.684 \mathrm{~g} / \mathrm{cm},{ }^{3}$ and the shear modulus $\mu$ of quartz is $2.947 \times 1011 \mathrm{~g} / \mathrm{cm} \mathrm{s}^{2}$. Around oscillation frequency of $7.995 \mathrm{MHz}$, a net change of $1 \mathrm{~Hz}$ corresponds to $1.34 \mathrm{ng}$ of materials adsorbed or desorbed onto the crystal surface of an area of $0.196 \mathrm{~cm}^{2}$. Gold coated quartz crystal electrodes were placed into ethanol and ultrasonically cleaned, then rinsed by de-ionized water. After dried with argon gas, Chitosan modified QCM electrodes were placed into the Teflon housing and the reference frequency

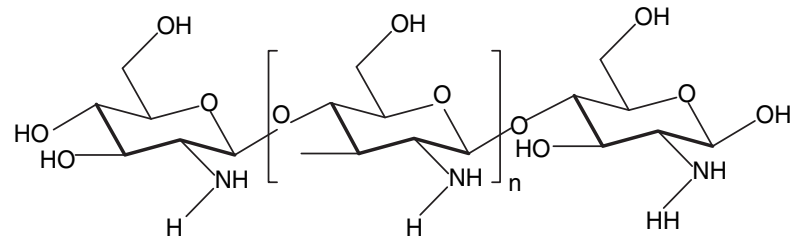

Fig. 1. Chemical formula of chitosan.

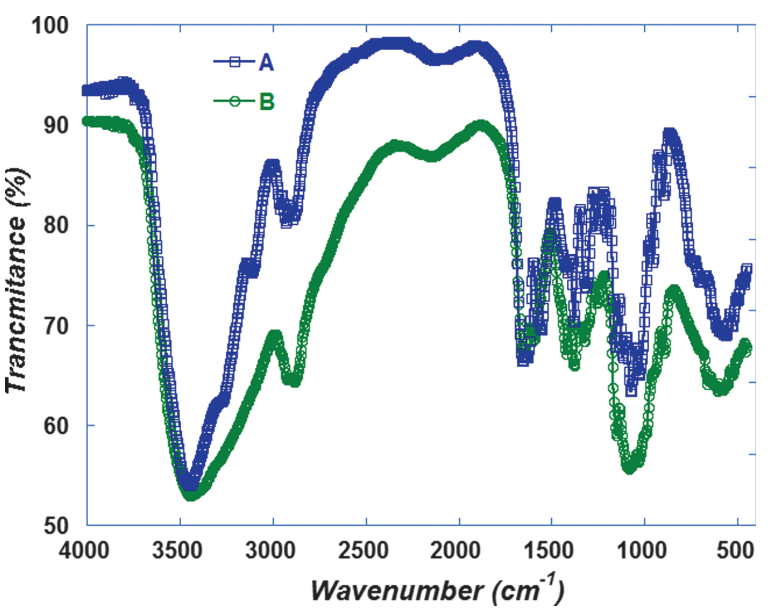

Fig. 2. FT-IR spectrum of chitin (A) and chitosan (B).

was recorded with unmodified one before the measurements. A partly filled closed container with saturated salt solutions generates a fixed level of relative humidity in the free room above the salt with good accuracy. The fixed point relative humidity level depends on the type of salt as given by Greenspan. ${ }^{22}$ The vapor molar concentration $\mathrm{C}(\mathrm{M})$ was calculated using ideal gas equation $(P V=$ $n R T)$ at the corresponding partial pressure for the relative humidity measured at the equilibrium point. ${ }^{18}$

The same experimental setup given in our previous work has been used to measure the QCM adsorption kinetics of chitosan films between $11 \% \mathrm{RH}$ and $94 \% \mathrm{RH}^{23}$ Here, the fixed value of $11 \% \mathrm{RH}$ is obtained inside a half-filled closed container with saturated $\mathrm{LiCl}$ and and $94 \% \mathrm{RH}$ with $\mathrm{K}_{2} \mathrm{SO}_{4}$ aquatic salt solutions. A hybrid system of QCM electrodes and a commercial humidity sensor was used for QCM measurements. Both signals coming from QCM and RH sensor were simultaneously recorded during adsorption process. For this purpose, a commercial EI-1050 digital relative humidity probe with a response time of $4.0 \mathrm{~s}$ and a resolution of $0.03 \% \mathrm{RH}$ was used with a USB controlled LabJack U12 ADC system combined with a single chip sensor module (SHT11) manufactured by Sensirion (Staefa, Switzerland).

\section{RESULTS AND DISCUSSION}

The fast response of Chitosan molecules to relative humidity changes is related with the hydrogen bonds formed with amine and hydroxyl groups described in Figure 3. The SEM image of chitosan film given in Figure 4 shows a relatively smooth surface morphology.

Figures 5(a) and (b) shows the frequency response of chitosan film covered on a gold coated QCM electrodes during adsorption process at fixed point relative humidity conditions between $11 \%$ and $94 \%$ RH. Figure 5(b) shows some cycles of QCM frequency response recovering back to the initial value after adsorption and desorption processes between $43 \% \mathrm{RH}, 75 \% \mathrm{RH}$ and $84 \% \mathrm{RH}$. 


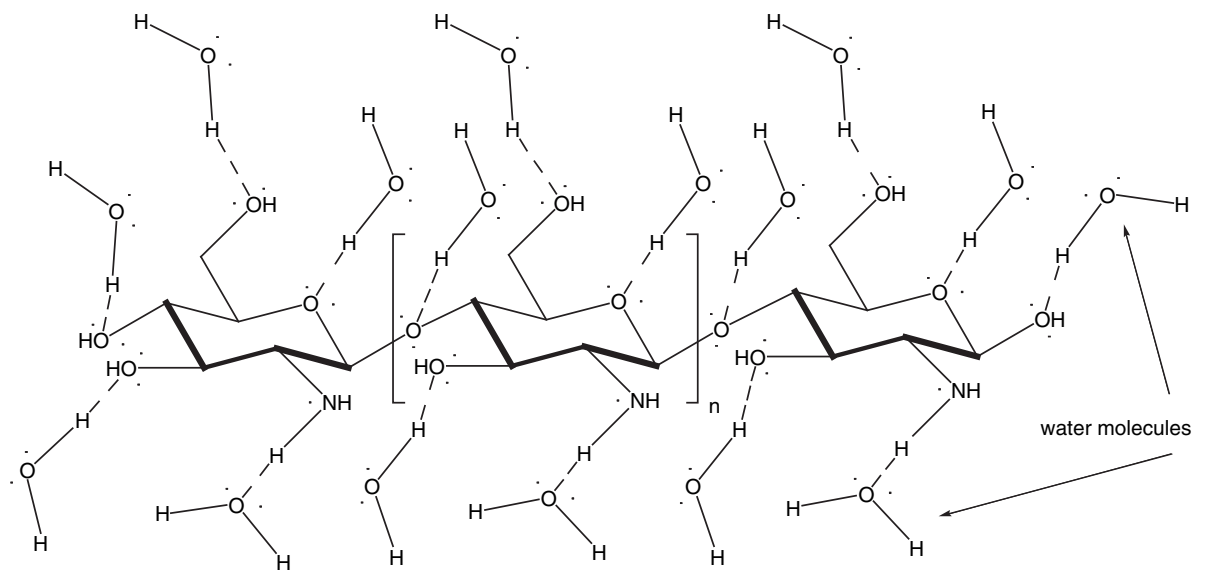

Fig. 3. Possible sites on a chitosan molecule to form $\mathrm{H}$ bonds with water.

Figure 5(a) represent the QCM response of chitosan to the increasing and decreasing relative humidity steps between $11 \%$ and $94 \% \mathrm{RH}$ for an equal time intervals to see if there is any residual adsorbed mass left on the chitosan film. About $10 \%$ frequency difference between $94 \%$ RH and $11 \%$ before and after adsorption and desorption process is the indication of a small hysteresis as a result of left resident absorbed water mass on the film. The QCM results show a linear dependence on the variation of relative humidity between $11 \%$ and $94 \% \mathrm{RH}$ as seen in Figure 5(c). Linear fitting to frequency shift versus \% RH is used to obtain the sensitivity of the chitosan structures. The sensitivity of the chitosan active layer is obtained as $7.5 \mathrm{~Hz} / \% \mathrm{RH}$.

To describe adsorption kinetics of gas molecules onto organic or inorganic films, Langmuir adsorption isotherm model is frequently used. ${ }^{24}$ According to this model, the rate of surface reaction to form a monolayer on the surface is given by

$$
\frac{d \theta}{d t}=k_{\mathrm{a}}(1-\theta) C-k_{\mathrm{d}} \theta
$$

$\theta$ is a unitless quantity, which express the fraction of surface coverage, $C$ is the gas concentration in the air, $k_{\mathrm{a}}$ and

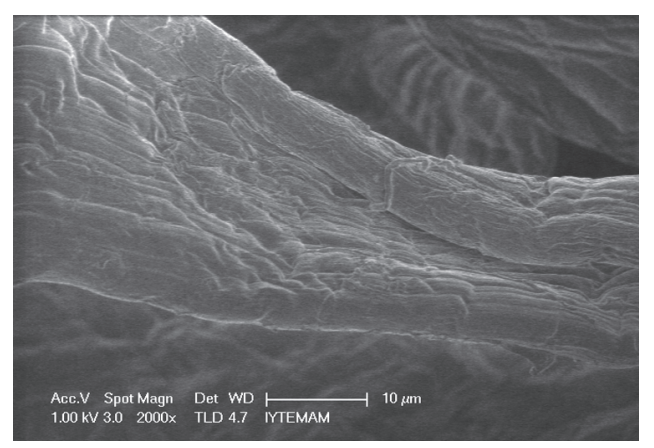

Fig. 4. SEM image of surface morphology of chitosan film.
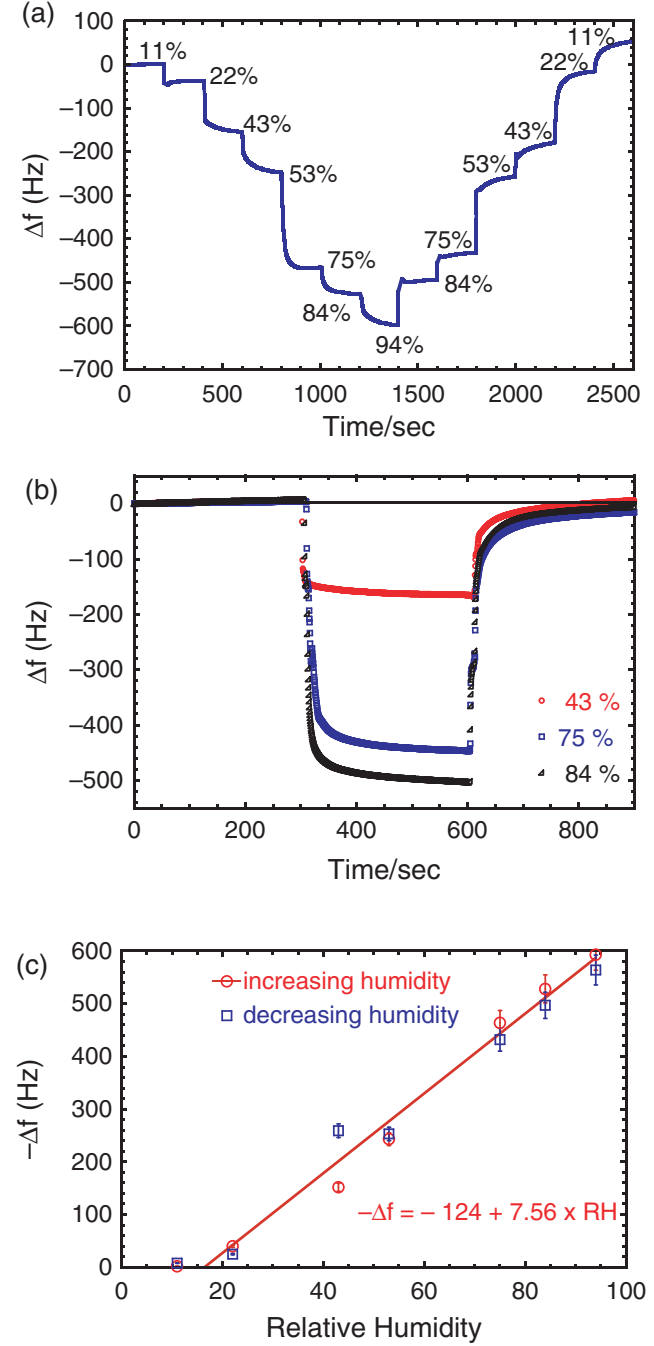

Fig. 5. QCM frequency shifts for adsorption and desorption cycles between $11 \%$ and $94 \%$ RH (a). QCM frequency shifts for various RH values as a function of time (b). The QCM adsorption and desorption data in Fig. 5a show a linear dependence on relative humidity (c). 
$k_{\mathrm{d}}$ are the adsorption and desorption constants, respectively. Integration of Eq. (2) leads to:

$$
\theta(t)=K^{\prime}\left(1-e^{-k_{\mathrm{obs}} t}\right)
$$

where $k_{\mathrm{obs}}$ is the inverse of the relaxation time and $K^{\prime}$ is the association constant defined as following;

$$
K^{\prime}=\frac{C}{C+\left(k_{\mathrm{d}} / k_{\mathrm{a}}\right)} \text { and } k_{\mathrm{obs}}=k_{\mathrm{a}} C+k_{\mathrm{d}}
$$

In this study, QCM has been used to measure the fractional coverage $\theta$ as a function of time during the adsorption of water vapor molecules by chitosan film, while the increase in the frequency shift rejects the molecular mass uptake or loss. Thus the difference between the oscillation frequency shift $(\Delta f)$ of coated and uncoated QCM is directly proportional to the adsorbed mass of moisture molecules. The relationship between the surface adsorption kinetics and frequency shift $(\Delta f)$ of QCM can be expressed as following;

$$
\frac{d \Delta f}{d t}=\left(\Delta f_{\max }-\Delta f\right) k_{\mathrm{a}} C-k_{\mathrm{d}} \Delta f
$$

where $\Delta f$ and $\Delta f_{\max }$ are the QCM resonance frequency shifts, $k_{\mathrm{a}}$ and $k_{\mathrm{d}}$ are the adsorption and desorption rate constants, $\mathrm{C}$ is the concentration of water molecules in air, $t$ is the time. To determine the partial pressure of water vapor at related temperature, the standard steam tables were used. The saturated salt solutions is a very adequate method to calibrate humidity sensors. ${ }^{25}$ Temperature was kept constant during the measurements around $20^{\circ} \mathrm{C}$. The vapor molar concentration $\mathrm{C}$ was calculated from the ideal gas equation $(P V=n R T)$ at the corresponding partial pressure for the relative humidity measured at the equilibrium point as shown by Okur et al. ${ }^{23}$

The time variation of the change in the frequency due to adsorption can be obtained as following,

$$
\Delta f(t)=\Delta f_{\max } K^{\prime}\left(1-e^{-k_{\mathrm{obs}} t}\right)
$$

Since the frequency shift is proportional with the change in the absorbed mass by the film on the QCM electrodes, the time dependent frequency shift can be fitted by Langmuir isotherm adsorption. From the curve fit, $k_{\mathrm{obs}}=$ $0.106 s^{-1}$ is obtained.

Using Sauerbrey relation given in Eq. (1), $(\Delta m=$ $-(1.34 n g / H z) \Delta f)$, the time dependent variation of mass of the adsorbed water vapor molecules on the chitosan film surface $\Delta m_{t}$ can be defined as following:

$$
\begin{gathered}
\Delta m_{t}=\Delta m_{\infty}\left(1-e^{-t / \tau}\right) \\
\tau^{-1}=k_{a}[\text { water vapour molecules }]+k_{\mathrm{d}}
\end{gathered}
$$

$\Delta m_{\infty}$ is the maximum amount of adsorbed humidity molecules on the surface for $t \rightarrow \infty$ and $\tau$ is the relaxation time. From the least square fit to Eq. (7), the relaxation time of the adsorption process is calculated as $9.5 \mathrm{~s}$. (Fig. 6)

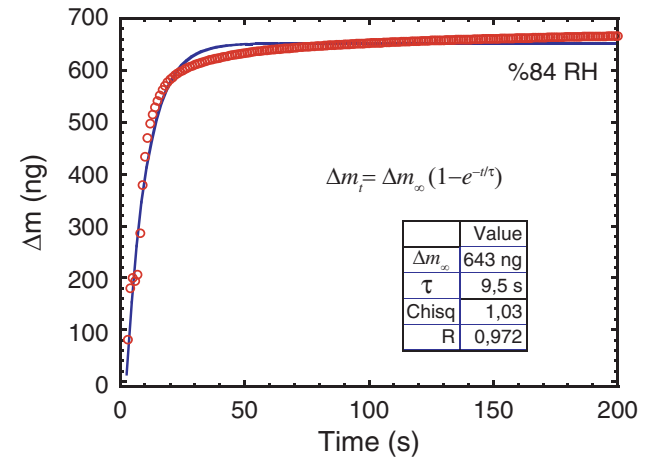

Fig. 6. The least square fit (solid line) to the Langmuir adsorption isotherm model given in Eq. (7) for the adsorbed water mass at $84 \% \mathrm{RH}$.

Figure 5 shows the least square fit (solid line) using the modified Langmuir adsorption isotherm model given in Eq. (7) for the adsorption parts of the data for \%84 $\mathrm{RH}$ given in Figure 5(b).

The average values of adsorption and desorption rates $\left(k_{\mathrm{a}}\right.$ and $k_{\mathrm{d}}$ ) between $11 \% \mathrm{RH}$ (concentration of $1.06 \times$ $10^{-4} \mathrm{M}$ ) and $84 \% \mathrm{RH}$ (concentration of $8.06 \times 10^{-4} \mathrm{M}$ ) were calculated as $149.10 \mathrm{M}^{-1} \mathrm{~s}^{-1}$ and $4.1 \times 10^{-4} \mathrm{~s}^{-1}$ respectively. The equilibrium constant, $K_{\text {equ }}$, for chitosan film is determined as following,

$$
K_{\text {equ }}=\frac{k_{\mathrm{a}}}{k_{\mathrm{d}}}
$$

The Gibbs free energy $\Delta G$ of adsorption/desorption process in terms of $K_{\text {equ }}$ at a constant temperature is defined as. $^{26}$

$$
\Delta G=-R T \ln K_{\mathrm{equ}}
$$

The Gibbs free energy for adsorption processes was calculated using Eq. (10). The $K_{\text {equ }}$ and Gibbs free energy for adsorption are obtained as $4.14 \times 10^{5}$ and $-12.93 \mathrm{~kJ} / \mathrm{mol}$, respectively. The negative sign shows that there is an energy loss for water molecules on chitosan film surface, since the water molecules are absorbed by the chitosan film surface during adsorption process. For a favored (spontaneous) reaction, the change in the Gibbs free energy should be negative $(\Delta G<0)$. Hence, the kinetic analysis for chitosan films shows a favored kinetic reaction with humidity molecules.

The Langmuir analysis shows that the adsorption rate of chitosan layer with functional amine and hydroxyl groups in the molecular structure is at least 15 times faster compare to the adsorption rates of organic calix[4]arene ${ }^{14}$ and organometallic Ruthenium ${ }^{15}$ derivatives with carboxylate and sulphonate groups.

The QCM results show that chitosan films are extremely sensitive to humidity and give reproducible adsorption results against to humidity changes for short time intervals, due to amine and hydroxyl groups in the molecular structure. 


\section{CONCLUSION}

Chitosan was synthesized by partially deactylation of chitin flakes with a high DD value, $89 \%$. QCM technique is used to investigate the response of chitosan film to relative humidity changes. We explained the adsorption kinetics under various fixed relative humidities between $11 \%$ and $94 \%$ by using Langmuir absorption model at $20{ }^{\circ} \mathrm{C}$ constant QCM temperature. The average Gibbs free energy for adsorption is obtained as $-12.93 \mathrm{~kJ} / \mathrm{mol}$ resulting that the chitosan films show a favored kinetic reaction against moist molecules in air. The QCM results show that chitosan can be used for humidity sensor applications and moisture adsorbent at large scale for industrial applications around room temperature.

Acknowledgments: We would like to thank to Ahmet Emin Eroğlu for supporting chitin and Ezel Boyac $\iota$ for her help during chitosan synthesis. This research was partially supported by TÜBİTAK (Turkish Scientific and Technological Research Council) under project number TBAG $109 \mathrm{~T} 240$.

\section{References and Notes}

1. L. James, Polymer Degradation and Stability 59, 145 (1998).

2. Y. Zhang, K. Yu, R. Xu, D. Jiang, L. Luo, and Z. Zhu, Sensors, and Actuators A: Physical 120, 142 (2005).

3. N. Wang, N. Zhang, and M. Wang, Computers and Electronics in Agriculture 50, 1 (2006).

4. T. Seiyama, N. Yamazoe, and H. Arai, Sensors and Actuators 4, 85 (1983).

5. P. G. Su, Y. L. Sun, and C. C. Lin, Sensors and Actuators B: Chemical 115, 338 (2006).
6. P. G. Su and S. C. Huang, Sensors and Actuators B: Chemical 113, 142 (2006).

7. P. G. C. Piseri, P. Milani, and G. A. Bongiorno, (2005).

8. H. Ito, S. Kakuma, R. Ohba, and K. Noda, 631, 635 (2004).

9. Y. Zhang, K. Yu, S. Ouyang, L. Luo, H. Hu, Q. Zhang, and Z. Zhu, Physica B: Condensed Matter 368, 94 (2005).

10. Y. K. Hong, H. K. Park, S. Q. Lee, K. S. Moon, and M. Levy, 140 (2004).

11. Y. Sakai, Y. Sadaoka, and M. Matsuguchi, Sensors and Actuators B: Chemical 35, 85 (1996).

12. C.-D. Feng, S.-L. Sun, H. Wang, C. U. Segre, and J. R. Stetter, Sensors and Actuators B: Chemical 40, 217 (1997).

13. K. G. Ong, C. Grimes, C. Robbins, and R. Singh, Sensors and Actuators A: Physical 93, 33 (2001).

14. S. Okur, M. Kus, F. Ozel, and M. Yilmaz, Sensors and Actuators B-Chemical 145, 93 (2010).

15. K. Ocakoglu and S. Okur, Sensors and Actuators B-Chemical 151, 223 (2010).

16. G. Sauerbrey, Z. Phys. 155, 206 (1959).

17. S. I. Ohira, E. Wanigasekara, D. M. Rudkevich, and P. K. Dasgupta, Talanta 77, 1814 (2009).

18. E. BoyacI, A. E. Eroglu, and T. Shahwan, Talanta 80, 1452 (2010).

19. J. Brugnerotto, J. Lizardi, F. Goycoolea, W. Argüelles-Monal, J. Desbrieres, and M. Rinaudo, Polymer 42, 3569 (2001).

20. A. L. Parize, M. Heller, V. T. Fávere, M. C. M. Laranjeira, I. M. C. Brighente, G. A. Mcke, and T. C. R. Souza, Lat. Am. J. Pharm. 28, 19 (2009).

21. Z. Dos Santos, A. Caroni, M. Pereira, D. da Silva, and J. Fonseca, Carbohydrate Research 344, 2591 (2009).

22. D. Ambrose and I. Lawrenson, The Journal of Chemical Thermodynamics 4, 755 (1972).

23. S. Okur, M. Kus, F. Özel, V. Aybek, and M. YIlmaz, Talanta 81, 248 (2010).

24. D. Karpovich and G. Blanchard, Langmuir 10, 3315 (1994).

25. Y. A. Cengel and M. A. Boles, Thermodynamics: An Engineering Approach; McGraw-Hill Higher Education, (2006).

26. S. Qiu, L. Sun, H. Chu, Y. Zou, F. Xu, and N. Matsuda, Thin Solid Films 517, 2905 (2009). 